\title{
In-Band and Out-Of-Band Distortions Optimization for ATSC 3.0 Transmission: A Novel TR PAPR Reduction Algorithm
}

\author{
$1^{\text {st }}$ Lahbabi Naila \\ Univ Rennes \\ INSA Rennes, CNRS, IETR-UMR6164 \\ F-35000 Rennes, France. \\ naila.lahbabi@insa-rennes.fr
}

\author{
$2^{\text {nd }}$ Jean-François Hélard \\ Univ Rennes \\ INSA Rennes, CNRS, IETR-UMR6164 \\ F-35000 Rennes, France. \\ jean-francois.helard@insa-rennes.fr
}

\author{
$3^{\text {rd }}$ Matthieu Crussière \\ Univ Rennes \\ INSA Rennes, CNRS, IETR-UMR6164) \\ F-35000 Rennes, France. \\ matthieu.crussiere@insa-rennes.fr
}

\begin{abstract}
This paper presents a novel Tone Reservation (TR) Peak to Average Power Ratio (PAPR) algorithm for the American digital video broadcasting (ATSC 3.0) standard. As any multicarrier communication systems, ATSC 3.0 transmitters suffer from high peaks of the transmitted signal. The high signal fluctuations constitute a major disadvantage of these systems. Indeed, they constraint the high power amplifiers to operate in their linear region which leads to poor spectral efficiency. To reduce the amplitude of the signal peaks, a TR gradient based algorithm has been proposed as an option within the ATSC 3.0 standard. Although TR-based algorithms have been intensively investigated these last years, very few today's transmitters implement these algorithms as they do not offer a high performance/complexity trade-off. This paper presents a novel TR-based algorithm, named as Grouped Carrier Peak Windowing (GCPW), which offers higher performance than the standard TR gradient-based algorithm while being implementable and compatible with the ATSC 3.0 standard. We also show through simulations that the proposed algorithm highly outperforms the gradient-based standard algorithm in terms of both in-band and out-of-band distortions. Results show that the novel algorithm offers very promising performance in terms of both modulation error rate and shoulder attenuation.
\end{abstract}

Keywords- ATSC 3.0, tone reservation, non-linear HPA, modulation error ratio, power spectral density, shoulder attenuation, OFDM, peak-to-average power ratio, in-band and out-of-band distortions.

\section{INTRODUCTION}

The American digital video broadcasting (ATSC 3.0) standard is a multicarrier communication system based on the Orthogonal Frequency Division Multiplexing (OFDM) modulation. OFDM modulation is widely employed by various multicarrier systems such as Digital Video Broadcast (DVB) and Long Term Evolution (LTE). However, it suffers as any multicarrier modulation from the high peaks in the time-domain of the transmitted signal, which is due to the summation of many subcarrier components via the Inverse Fast Fourier Transform (IFFT). Consequently, ATSC 3.0 transmitters suffer from Peakto-Average-Power-Ratio (PAPR).

Several PAPR reduction techniques have been proposed to reduce the transmitted signal fluctuations in order to maximize the power efficiency of High Power Amplifiers (HPA). In fact, these essential component in any modern communication systems are inherently Non-Linear (NL). The power efficiency is maximized when the HPA is operating near its NL region but to the detriment of severe in-band and out-of-band distortions. The NL characteristics of HPA cause amplitude and phase distortions to high PAPR signals. To deal with this issue, the HPA should operate in its linear region which degrades its power efficiency.

PAPR reduction of OFDM signals has been the focus of many studies summarized in [1] [2] [3]. Among the most widely known PAPR reduction techniques, clipping and filtering [4], coding [5], partial transmit sequence [6] and selected mapping [7]. However, the gain of such techniques is limited due to their low performance/complexity trade-off and the necessity, for some of them, to transmit side information.

Recently, two PAPR reduction techniques named as Tone Reservation (TR) [8] and Active Constellation Extension (ACE) [9] have been adopted as an alternative solution by many standards such as DVB-second generation (DVB-T2) [10], DVB for Next Generation Handled (DVB-NGH) and recently by the ATSC 3.0 standard [11]. The TR concept is based on the allocation of a subset of subcarriers called as Peak Reserved Tones (PRTs) for PAPR reduction purposes. The main idea behind the TR concept is the optimization of the complex values to load on these PRTs to lower the PAPR of the transmitted signal. In fact, the time-domain kernel obtained from these complex values is added to the original one and the resulting signal is expected to have lower PAPR. The optimal solution is originally presented as a convex optimization problem but can easily be transformed to a linear problem. In DVB-T2, DVB-NGH and ATSC 3.0 standards, the TR concept is presented as an iterative gradient-based algorithm and the computation of the optimal complex values assigned to the PRTs is performed iteratively. At each iteration, the highest peak of the time-domain signal is detected. The algorithm generates a shifted copy of the kernel such as it coincides with the detected peak position. Then, the kernel amplitude and phase are adjusted to lower the peak of the resulting signal. When the maximal number of iterations is reached or when all the signal peaks are below a predefined threshold $V_{\text {clip }}$, the algorithm exits. This TR-based algorithm is detailed in [10] for DVB-T2 and DVB-NGH standards and in [11] for the ATSC 3.0 standard. Unfortunately, it does not offer a sufficient performance/complexity trade-off to be implemented in todays modulators. 
Recently, several alternatives to the gradient-based TR algorithm have been investigated. A new kernel definition has been proposed in [12] along with a new TR-based algorithm named as Individual Carrier Multiple Peaks (ICMP). It is based on a comb-like kernel generated by activating a single PRT per iteration and targeting the reduction of multiple signal peaks at a time. In [13], a very promising TR-based algorithm named as Grouped Individual Carrier for Multiple Peaks (GICMP) has been proposed for DVB-T2 standard. This work presents an ameliorated version of the ICMP algorithm. The new proposed kernel is now obtained by activating a sub-group of the PRTs at each iteration and offers very good performance with low complexity and is implementable in the DVB-T2 and ATSC 3.0 standards. In [14], the authors proposed an implementationfriendly algorithm named as Grouped Carrier Peak Windowing (GCPW) implementable in DVB-T2 and ATSC 3.0 standards. This novel algorithm defines a new method to detect the transmitted signal peaks which leads to lower hardware resources such as latency, complexity and memory.

GCPW aims at reducing only the signal peaks above a precomputed and optimized threshold. Hence, the OFDM timedomain samples can be processed when they are received without the necessity of storage and sorting operations as with the GICMP algorithm. This highly reduces the algorithm complexity, latency and memory requirements. The optimization of the GCPW algorithm parameters such as the number of PRT groups and the maximal number of targeted peaks enables very promising performance compared to the gradient-based algorithm. In this paper, we study the in-band and out-of-band distortions of the GCPW algorithm for the ATSC 3.0 standard. We optimize the in-band distortion represented by the Modulation Error Rate (MER) by investigating the optimal GCPW parameters. Then, we study the effect of GCPW on the Power Spectral Density (PSD) of the transmitted amplified signal. We show via simulation that the proposed implementation-friendly GCPW algorithm offers very good performance in terms of inband and out-of-band distortions with limited complexity and implementation resources requirements in both $8 \mathrm{~K}$ and $32 \mathrm{~K}$ modes which are the preferred modes for mobile and fixed reception, respectively.

The remaining of this paper is organized as follows: Section II recalls the OFDM system model and some issues related to PAPR reduction. Section III reminds the in-band and out-ofband distortions definitions. In Section IV we present the state of the art of TR solutions. In Section V, the GCPW algorithm is described and the threshold computation is detailed. MER, PSD and shoulder attenuation performances of the GCPW algorithm are evaluated in Section VI and conclusions are drawn in Section VII.

\section{OVERVIEW OF OFDM SYSTEM MODEL AND PAPR RELATED ISSUES}

\section{A. OFDM System Model}

The ATSC 3.0 standard considers OFDM modulation where the continuous-time baseband signal is transmitted over $N$ subcarriers and expressed as

$$
x(t)=\mathcal{F}\{\mathbf{X}\}=\frac{1}{\sqrt{N}} \sum_{k=0}^{N-1} X_{k} e^{j 2 \pi \frac{k t}{N T}}, 0 \leq t<N T,
$$

$\mathrm{X}$ represents a sequence of complex symbols $\left[X_{0}, \ldots, X_{n}, \ldots, X_{N-1}\right], T_{u}=N T$ is the useful part of the OFDM symbol duration and $T$ is the sampling period. $\mathcal{F}$ is the OFDM modulation function and $j=\sqrt{-1}$. Classically, to avoid Inter Symbol Interference (ISI) in the useful part, a guard interval of duration $\Delta$ is inserted between adjacent OFDM symbols and the total OFDM symbol duration is then $T_{s}=T_{u}+\Delta$. With ATSC 3.0 standard, as in most of the OFDM systems called Cyclic Prefix OFDM (CP-OFDM), the guard interval is a cyclic extension of each OFDM symbol.

\section{B. HPA Model}

The HPA model considered in this paper is the well-known Rapp model [15], commonly used to model solid-state HPAs in broadcasting systems. At its output, the equivalent continuoustime baseband signal is given as

$$
y(t)=\frac{x(t)}{\sqrt[2 p]{1+\left(\frac{|x(t)|}{v_{s a t}}\right)^{2 p}}} .
$$

where $x(t)$ is the input signal and $p$ the knee factor which reveals the smoothness of transition from the linear region to the saturation region of HPA.

It is well known that the input power of an HPA must be reduced by a a value called the input back-off (IBO) to achieve undistorted amplified signal $y(t)$. This value represents the difference in $\mathrm{dB}$ between the carrier input power at the operation point and the saturation point, as given below

$$
\mathrm{IBO}=10 \cdot \log _{10}\left(\frac{v_{\text {sat }}^{2}}{\mathbb{E}\left[|x(t)|^{2}\right]}\right),
$$

where $v_{\text {sat }}$ is the saturation voltage of the HPA and $\mathbb{E}[$.$] is the$ expectation operator.

\section{PAPR definition}

The PAPR of $x(t)$, over a symbol period $T_{s}$, is a random variable which measures the ratio of the signal maximum instantaneous power to its mean power and is defined by

$$
P A P R_{x(t)}=\frac{\|x(t)\|_{\infty}^{2}}{\frac{1}{T_{s}} \int_{0}^{T_{s}}\|x(t)\|_{2}^{2} \cdot d t},
$$

where $\|\cdot\|_{\infty}$ and $\|\cdot\|_{2}$ denote infinity and Euclidean norms, respectively.

\section{IN-BAND AND OUT-OF-BAND DISTORTIONS}

In this paper, we evaluate the performance of the proposed TR-based algorithm in terms of in-band and out-of-band distortions. The well-known figure of merit characterizing in-band distortions is the MER. To evaluate the out-of-band distortions, we study the PSD of the transmitted signal to measure the shoulder attenuation which characterizes the linearity of the OFDM signal without reference to a spectrum mask. The spectral mask is a crucial linearity requirement used to ensure that the transmitter does not interfere with the spectrum of neighboring channels. 


\section{A. MER metric}

In the broadcasting industry, the modulation error ratio (MER) is the most widely used figure of merit for system performance and is defined in $\mathrm{dB}$ as

$$
\operatorname{MER}\{\mathbf{X}, \widehat{\mathbf{X}}\}=10 \log _{10}\left(\frac{\|\mathbf{X}\|_{2}^{2}}{\|\mathbf{X}-\widehat{\mathbf{X}}\|_{2}^{2}}\right),
$$

where $\mathbf{X}$ is the ideal symbol vector measured at the input of the amplifier and $\widehat{\mathbf{X}}$ is measured at the output of the HPA. In literature, the Complementary Cumulative Density Function (CCDF), which depends on the amplitude of the highest signal peak, is the most known parameter for PAPR analysis. However, MER measures the energy of the distortion induced by the NL effects of the HPA which is related in timedomain to the energy above a given threshold. MER metric depends on the energy of the time-domain OFDM signal above this threshold and is very useful for the good dimensioning of the signal.

\section{B. PSD metric}

The OFDM symbols constitute a juxtaposition of equallyspaced orthogonal carriers. The power spectral density $P_{k}(f)$ of the transmitted signal at frequency

$$
f_{k}=f_{c}+\frac{k}{T_{u}} \quad \text { for } \quad-\frac{N-1}{2} \leq k \leq \frac{N-1}{2}
$$

with $f_{c}$ the central frequency, is defined by the following expression

$$
P_{k}(f)=\left[\frac{\sin \pi\left(f-f_{k}\right) T_{s}}{\pi\left(f-f_{k}\right) T_{s}}\right]^{2},
$$

with $T_{s}=T_{u}+\Delta$. Classically, the intercarrier spacing is equal to $\frac{1}{T_{u}}$ but, as CP-OFDM is used, the power spectral density of each carrier of the transmitted signal is null at a distance equal to $\pm \frac{1}{T_{s}}$ of the frequency corresponding to its maximum. The overall power spectral density of the modulated data is the sum of the power spectral densities of all the carriers as

$$
P(f)=\sum_{k=-\frac{N-1}{2}}^{\frac{N-1}{2}}\left[\frac{\sin \pi\left(f-f_{k}\right) T_{s}}{\pi\left(f-f_{k}\right) T_{s}}\right]^{2},
$$

\section{TONE RESERVATION BASED ALGORITHMS FOR PAPR REDUCTION}

\section{A. TR Concept}

In [8], Tellado introduced the TR concept which is based on the dedication of a subset of PRTs for PAPR reduction purposes. A kernel signal generated using the subset of PRTs is added in time-domain to the original one in order to lower its PAPR. Let us consider that $R$ subcarriers are allocated for PAPR reduction of the OFDM system of $N$ subcarriers. We define $\mathcal{B}$ as the PRT subset of these $R$ locations and $\mathbf{C}$ as the vector of $R$ peak reduction symbols transmitted on these positions and zeros elsewhere. Similarly, let the complement set $\mathcal{B}^{c}$ be the Data Tone (DT) subset of the useful data positions and $\mathbf{D}$ the vector of the $N-R$ associated transmitted data symbols and zeros elsewhere. The DT and PRT sets are totally disjoint, i.e. $\mathcal{B} \cap \mathcal{B}^{c}=\emptyset$. Since at the receiver side only data tones are considered to recover the transmitted data, no side information is needed in TR concept.

In frequency and time domains, the transmitted signal is then expressed as

$$
\mathbf{X}=\mathbf{C}+\mathbf{D} \quad \stackrel{\mathcal{F}}{\Longleftrightarrow} \quad \mathbf{x}=\mathbf{c}+\mathbf{d},
$$

where

$$
\mathbf{C}=\left\{\begin{array}{l}
C_{k} \neq 0, \quad \forall k \in \mathcal{B}, \\
C_{k}=0, \quad \forall k \in \mathcal{B}^{c},
\end{array}\right.
$$

and

$$
\mathbf{D}=\left\{\begin{array}{l}
D_{k} \neq 0, \quad \forall k \in \mathcal{B}^{c} \\
D_{k}=0, \quad \forall k \in \mathcal{B}
\end{array}\right.
$$

\section{B. Optimal TR solution}

The main issue of the TR concept is the computation, for each OFDM symbol, of the adequate complex values $C_{k}$ to load on the reserved tone in order to lower the PAPR. Such problem can be formulated as a Quadratically Constrained Quadratic Program (QCQP) [16]

$$
\begin{array}{cl}
\min _{\mathbf{C}} & \Lambda=\left\|\mathbf{d}+\mathbf{F}_{N}^{H} \mathbf{C}\right\|_{\infty}^{2} \\
\text { subject to, } & \|\mathbf{C}\|_{\infty}^{2} \leq \frac{\Gamma}{N-R}\|\mathbf{D}\|_{2}^{2},
\end{array}
$$

where $\mathbf{F}_{N}$ is the Fourier matrix of size $N$ and $\Gamma=10^{\frac{\lambda}{10}}$ is the power level gap with $\lambda$ the difference in $\mathrm{dB}$ between the maximum PRT power and the mean DT power. Note that the power constraint imposed on the reserved tones is a peak power constraint defined with respect to the power level associated to data tones. This corresponds to the case of DVBT2, DVB-NGH and ATSC3.0 specifications, where $\lambda=10 \mathrm{~dB}$ maximum.

QCQP problem can also be viewed as a special case of Second Order Cone Program (SOCP) [17], which is a convex optimization problem class that minimizes a linear function over the intersection of an affine set and the product of secondorder (quadratic) cones. Finding the optimal solution using SOCP or QCQP requires very high computational complexity which completely prevents the implementation of such algorithms in practical transmitters.

\section{TR Gradient-Based algorithm for the ATSC 3.0 standard}

The ATSC 3.0 standard defines an iterative TR-based algorithm to compute the complex values $C_{k}$ loaded on the PRTs locations. Originally proposed in [18], this solution defines impulse-like kernels to iteratively cancel the signal peaks. The reference kernel signal denoted as $\kappa$, is a Dirac-like pulse obtained by letting $C_{k}=1, \forall k \in \mathcal{B}$, that is

$$
\boldsymbol{\kappa}=\mathcal{F}\left\{\mathbf{1}_{T R}\right\}, \quad \text { where, } \mathbf{1}_{T R}= \begin{cases}1, & k \in \mathcal{B}, \\ 0, & k \in \mathcal{B}^{c} .\end{cases}
$$

The proposed kernel aims at reducing the signal peak by shifting the original kernel $\kappa$ to make its peak position coincide with the detected one. Hence, the QCQP optimization problem can be restated as follows 


$$
\begin{aligned}
\min _{\rho_{i}, \tau_{i}} & \Lambda=\left\|\mathbf{d}+\boldsymbol{\kappa} \circledast \sum_{i=1}^{N_{i}} \rho_{i} \boldsymbol{\delta}_{N}\left[n-\tau_{i}\right]\right\|_{\infty}^{2} \\
\text { subject to, } & \|\mathbf{C}\|_{\infty}^{2} \leq \frac{\Gamma}{N-R}\|\mathbf{D}\|_{2}^{2},
\end{aligned}
$$

in which $\circledast$ stands for circular convolution and $\boldsymbol{\delta}_{N}[n]$ is the Dirac delta discrete sequence vector of size $N$. The optimal solution consists in finding the optimal time delays $\tau_{i}$ and weigthing factors $\rho_{i}$, for a sufficient number of iterations $N_{i}$ which leads to identical results as the QCQP algorithm. However, a suboptimal approach can be followed, by fixing $\Lambda$ to a fixed reference value $\Lambda$ corresponding to the average value obtained in simulation by running the optimal solution. Then, an iterative process can be established consisting in detecting and cancelling the first highest peak, and then repeating the process iteratively for the other peaks. The algorithm exits as soon as the power constraint is violated or when a predefined maximum number of iterations is reached.

In the following, this algorithm is named as Time Tracking Power Control (TTPC). This TTPC algorithm suffers from two major issues that makes it very difficult to be implemented in todays ATSC 3.0 transmitters. First its very slow convergence which results in a high number of iterations. Second, the available amount of power dedicated to the reserved tones is inefficiently exploited. That is why, multiple researches have been focusing these last years on enhancing the performance of TR gradient-based algorithms (e.g. [19], [20]). However, even if some improvements have been achieved, the proposed solutions does not sufficiently alleviate the convergence and power constraint issues for practical implementation.

\section{New Kernel Definition fOR TR-BASEd PAPR REDUCTION ALGORITHM}

In this section, we introduce a new kernel definition and power allocation to the PRTs to benefit from all the amount of power available for PAPR reduction. We present a novel TRbased algorithm named as Grouped Carrier Peak Windowing (GCPW) which offers a good performance/complexity tradeoff. This algorithm is based on a grouping strategy of the PRTs into $G$ groups as follows

$$
\mathcal{B}=\left\{\mathcal{B}_{1}, \ldots, \mathcal{B}_{G}\right\}
$$

with,

$$
\mathcal{B}_{i}=\left\{\mathcal{P}_{i, j}, \quad 1 \leq i \leq G, \quad 1+\frac{(i-1) R}{G} \leq j \leq 1+\frac{i R}{G}\right\},
$$

where $\mathcal{P}_{i, j}$ is the $j^{\text {th }}$ tone in $\mathcal{B}_{i}$. At each iteration of the algorithm, a comb-like kernel is generated by activating a group of reserved tones. The total number of iterations is now equal to the number of PRT groups denoted here by $G$. The proposed kernel at the $i^{\text {th }}$ iteration is then defined as

$$
\boldsymbol{v}_{i}=\mathcal{F}\left\{\mathcal{B}_{i}\right\}
$$

with,

$$
\mathcal{B}_{i}= \begin{cases}\mathcal{P}_{i, j}=1, & 1+\frac{(i-1) R}{G} \leq j \leq 1+\frac{i R}{G} \\ \mathcal{P}_{i, j}=0, & \text { otherwise. }\end{cases}
$$

Without the use of any additional IFFT, the time-domain kernel is computed as

$$
v_{i}(n)=\sum_{j=1}^{\frac{R}{G}} p_{i, j}(n), \quad 1 \leq i \leq G,
$$

where,

$$
p_{i, j}(n)=\frac{1}{N} e^{-\jmath \frac{2 \pi \mathcal{P}_{i, j} n}{N}}, \quad 0 \leq n<N,
$$

is the kernel associated to the $\mathcal{P}_{i, j}$ PRT. The new optimization problem is now stated as follows

$$
\begin{aligned}
& \min _{\rho_{i, j}, \phi_{i, j}}\left\|\mathbf{d}+\sum_{i=1}^{G} \sum_{j=1}^{\frac{R}{G}} \rho_{i, j} e^{-\jmath \phi_{i, j}} \boldsymbol{p}_{i, j}\right\|_{\infty}^{2}, \\
& \text { subject to, }, \quad \rho_{i, j} \leq \frac{\Gamma}{N-R}\|\mathbf{D}\|_{2}^{2} .
\end{aligned}
$$

From this definition, we can easily understand that the power constraint is implicitly controlled via $\rho_{i, j}$. The optimal solution consists in an intensive search of the optimal values $\rho_{i, j}$ and $\phi_{i, j}$. However, after running the original QCQP algorithm, it turns out that almost all reserved tones should be loaded at the maximum allowed power level $A_{\text {max }}$. Hence, a simplified suboptimal problem can be obtained by fixing $\rho_{i, j}=A_{\max }, \forall i, j$. Additionally, the computation of the optimal $\phi_{i, j}$ values can also be simplified. The infinite norm can be converted into a quadratic one, by restricting the computation upon a given subset of the highest peak values of the OFDM time-domain signal. Hence, instead of minimizing the maximum peak amplitude (infinite norm criterion), we rather minimize the energy carried by a set $\mathcal{S}$ of multiple highest peaks.

The search of the subset $\mathcal{S}$ of multiple highest peaks can be further optimized. In fact, the detection a $\mathcal{S}$ of multiple highest peaks requires storage of the whole OFDM time-domain signal in addition to a sorting operation which increases the global latency. To overcome this issue, we propose to select at each iteration $i$, the first subset $\mathcal{S}_{i}$ of signal peaks above a given threshold $\tilde{\lambda}$. As in the gradient-based algorithm, such threshold can be optimized and pre-calculated through simulations. In addition, to limit the size of storage of these peaks, we keep only $\operatorname{Card}\left\{\mathcal{S}_{i}\right\} \leq S$ peaks for the phase computation, where $S$ is the maximum number of peaks. For the proposed GCPW algorithm, the sub-optimal problem is restated as follows

$$
\min _{\phi_{i, j}}\left\|\mathbf{d}\left(\mathcal{S}_{i}\right)+\mathbf{p}_{i, j}\left(\mathcal{S}_{i}\right) \cdot A_{\max } e^{-\jmath \phi_{i, j}}\right\|_{2}^{2},
$$

subject to, $\mathcal{S}_{i}=\left\{k:\left|d(k)+\sum_{j=1}^{i-1} c^{j}(k)\right|>\tilde{\lambda}\right\}$,

$$
\text { with, } \quad S_{i}=\operatorname{Card}\left\{\mathcal{S}_{i}\right\} \leq S,
$$

where $\mathcal{S}_{i}$ is the instantaneous subset of time-domain peak samples considered at each iteration, $c^{j}(k)$ is the kernel samples at iteration $j$ and $\tilde{\lambda}$ an amplitude threshold optimized through simulations as in the gradient-based case.

The optimal phase $\phi_{i, j}$ for each comb-like kernel $\boldsymbol{p}_{i, j}$, is computed taking into account several peak positions (hence the algorithm name). An iterative sub-optimal solution can be used to solve the problem via parallel computation of the optimal phases $\phi_{i, j}$. Assuming a set $\mathcal{S}_{i}$ of highest peaks of the signal and a kernel $\boldsymbol{p}_{i, j}$, the optimal phase $\phi_{i, j}^{*}$ at the $i^{\text {th }}$ iteration is obtained by solving the following sub-problem

$$
\min _{\phi_{i, j}}\left\|\mathbf{d}\left(\mathcal{S}_{i}\right)+\boldsymbol{p}_{i, j}\left(\mathcal{S}_{i}\right) \cdot A_{\max } e^{-\jmath \phi_{i, j}}\right\|_{2}^{2},
$$


and the optimal phase $\phi_{i, j}^{*}\left(\mathcal{S}_{\rangle}\right)$can then be expressed as

$$
\phi_{i, j}^{*}\left(\mathcal{S}_{i}\right)=\operatorname{atan}\left(\frac{\mathfrak{I m}\left\{\mathbf{d}^{\mathcal{H}}\left(\mathcal{S}_{i}\right) \boldsymbol{p}_{i, j}\left(\mathcal{S}_{i}\right)\right\}}{\mathfrak{R e}\left\{\mathbf{d}^{\mathcal{H}}(\mathcal{S}) \boldsymbol{p}_{i, j}\left(\mathcal{S}_{i}\right)\right\}}\right)-\frac{\pi}{2} .
$$

The new signal resulting from an original OFDM time-domain signal $\mathbf{d}$ after applying GCPW on $R$ reserved tones yields the following symbol vector :

$$
\mathbf{x}=\mathbf{d}+\mathbf{c} \quad \text { with, } \mathbf{c}=A_{\max } \sum_{i=1}^{G} \sum_{j=1}^{\frac{R}{G}} \boldsymbol{p}_{i, j} e^{-\jmath \phi_{i, j}^{*}\left(\mathcal{S}_{i}\right)}
$$

where $\boldsymbol{p}_{i, j}$ is obtained from (15) and $\phi_{i, j}^{*}\left(\mathcal{S}_{i}\right)$ is iteratively computed from (17).

Threshold computation: The threshold $\tilde{\lambda}$ is calculated as follows

$$
\tilde{\lambda}=\beta . A_{\text {mean }}, \beta \in \mathbb{R}_{+},
$$

with $\beta$ a positive multiplicative factor and $A_{\text {mean }}$ the mean value of the OFDM signal over $M$ defined as

$$
A_{\text {mean }}=\frac{1}{M \cdot N} \sum_{m=1}^{M} \sum_{n=1}^{N}\left|d_{m}(n)\right|
$$

where $\left|d_{m}(n)\right|$ are the absolute values of all time samples of the $m^{\text {th }}$ symbol.

To maximize the MER, the number of groups and the threshold value should be optimized. Hence, $\tilde{\lambda}$ value should be wisely optimized in order to select the most significant peaks.

\section{Simulation Results}

In this section, we evaluate the GCPW algorithm performance in terms of MER and shoulder measurements. We consider the following parameters: the HPA Rapp model with a knee factor $p=6$ and $v_{\text {sat }}=1,64$ QAM constellation and the ATSC 3.0 standard in $8 \mathrm{~K}$ and $32 \mathrm{~K}$ modes. As already mentioned, CP-OFDM is used in the ATSC 3.0 standard. For those simulations, the duration of the guard interval is $\Delta=$ $192 T$ in $8 \mathrm{~K}$ and $32 \mathrm{~K}$ modes. The ATSC 3.0 guidelines are summarized in the following.

\section{A. TR guidelines in ATSC 3.0}

In the ATSC 3.0 standard, the percentage of the subcarriers dedicated for PAPR reduction and denoted by $\kappa$ is fixed as $1 \%$. The number of reserved tones for PAPR reduction for different OFDM symbol sizes is given in Table I. Furthermore, the power boost of the PRTs compared to the DTs subcarriers is equal to $10 \mathrm{~dB}$. On this basis, the number of active subcarriers in ATSC 3.0, denoted by $N o C$, is given by the following expression

$$
N o C=N o C_{\max }-C_{\text {red-coeff }} \times C_{\text {unit }},
$$

where $N o C_{\max }$ is the maximum number of subcarriers in a symbol and $C_{u n i t}$ is a control unit factor taking a value of

TABLE I: Size of $R$ for different modes in ATSC 3.0.

\begin{tabular}{|c|c|c|c|}
\hline MODE & $8 \mathrm{~K}$ & $16 \mathrm{~K}$ & $32 \mathrm{~K}$ \\
\hline$N$ & 8192 & 16384 & 32768 \\
\hline$R$ & 72 & 144 & 288 \\
\hline
\end{tabular}

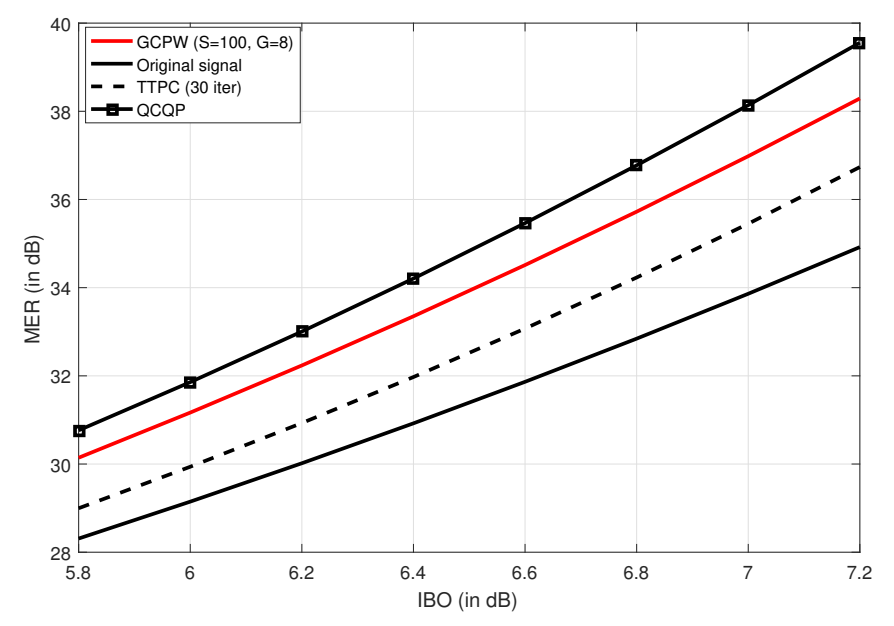

Fig. 1: MER with GCPW $(G=8, S=100)$ for: ATSC 3.0, 8K mode, 64 QAM and Rapp model HPA with $p=6$.

96 and 384 for $8 \mathrm{~K}$ and $32 \mathrm{~K}$ modes, respectively. $C_{\text {red-coef } f}$ is a positive integer ranging from 0 to 4 and indicating the number of carriers to be reduced. Considering $C_{\text {red-coeff }}=$ 0 , the $N o C$ value is 6913 for $8 \mathrm{~K}, 13285$ for $16 \mathrm{~K}$ and 27649 for $32 \mathrm{~K}$. The occupied bandwidth is $5.83 \mathrm{MHz}$ and the symbol rate $\frac{1}{T}$ is equal to $6.91 \mathrm{MHz}$.

\section{B. MER performance of the GCPW algorithm}

In $8 \mathrm{~K}$ and $32 \mathrm{~K}$ modes, the GCPW parameters have been optimized taking into account the MER measurements. In $8 \mathrm{~K}$ mode, the optimal GCPW parameters maximizing the MER are $G=8$ and $S=100$. The MER with the GCPW algorithm is shown in Fig. 1 and is compared to the original signal and to the optimal solution QCQP as well as to the standard TTPC algorithm. As we can notice, GCPW with $G=8$, i.e. 8 iterations outperforms the original signal and TTPC even after 30 performed iterations. At an IBO $=6.4 \mathrm{~dB}$, GCPW offers $33.35 \mathrm{~dB}$ MER and outperforms the original signal and the TTPC algorithm by $2.43 \mathrm{~dB}$ and $1.38 \mathrm{~dB}$, respectively. GCPW lags the QCQP solution by merely $0.86 \mathrm{~dB}$. Note that the optimal QCQP solution is far much too complex to be implemented and is given here as reference. In this case, GCPW needs only the storage of $S=100$ values without any sorting operation and offers very good performance/complexity trade-off.

In $32 \mathrm{~K}$ mode, the optimal GCPW parameters which maximize the MER are $G=12$ and $S=200$. We compare the MER performance of GCPW to the original signal and to QCQP and TTPC algorithms in Fig. 2. Here, GCPW needs 12 iterations and outperforms TTPC even if this one performs 30 iterations. The QCQP MER is also given as reference. As we can easily notice, GCPW offers very good MER performance. At IBO = $6.4 \mathrm{~dB}$, GCPW offers $33.21 \mathrm{~dB}$ and a gain of $2.33 \mathrm{~dB}$ and 1.48 $\mathrm{dB}$ compared to the original signal and TTPC, respectively. GCPW lags the optimal QCQP solution by less than $1 \mathrm{~dB}$. Hence, GCPW offers very promising results in terms of inband distortions with reduced complexity since it only requires 12 iterations and the necessity to store only 200 signal samples in $32 \mathrm{~K}$ mode. 


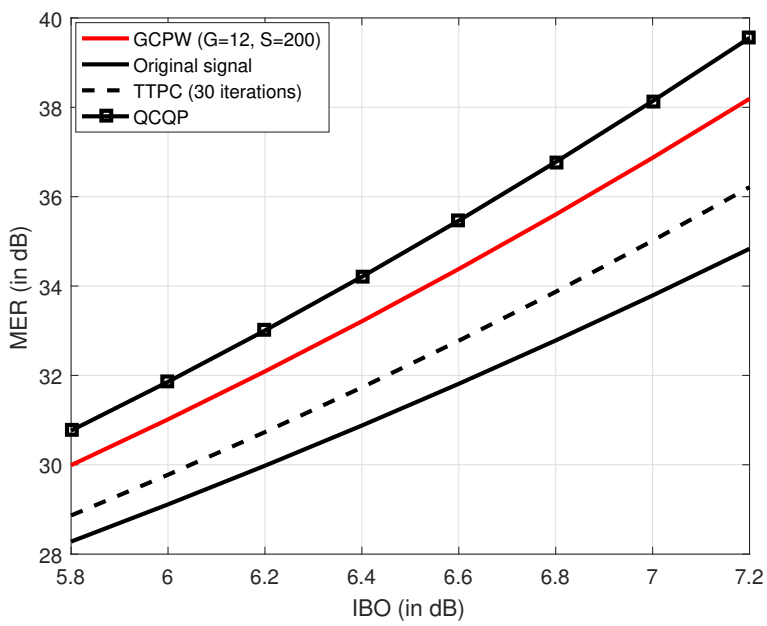

Fig. 2: MER with GCPW $(G=12, S=200)$ for: ATSC 3.0, $32 \mathrm{~K}$ mode, 64 QAM and Rapp model HPA with $p=6$.

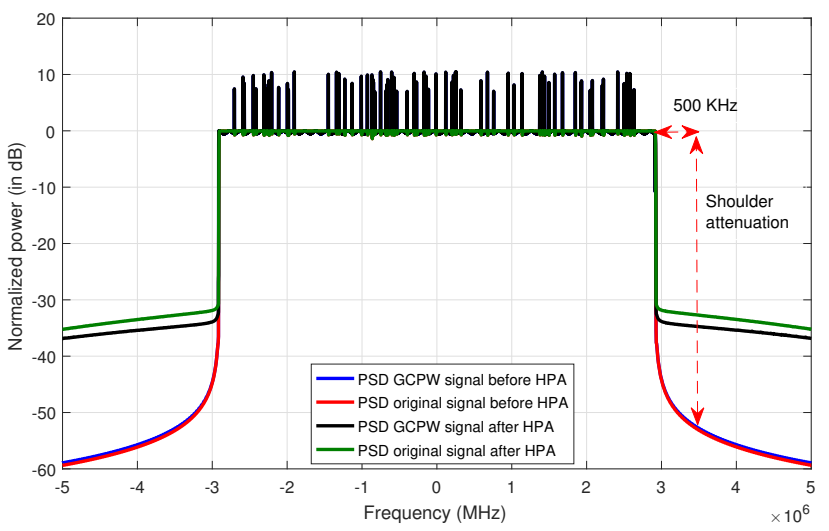

Fig. 3: PSD of original signal and transmitted signal with GCPW $(G=8, S=100)$ for: ATSC 3.0, 8K mode, 64 QAM and Rapp model HPA with $p=6, \mathrm{IBO}=6.4 \mathrm{~dB}$.

\section{PSD of the transmitted signal with GCPW algorithm}

In this section, we evaluate the PSD of the transmitted signal before and after the power amplification. After the HPA, the PSD of the original signal and with GCPW is given as an example at an IBO operation point of $6.4 \mathrm{~dB}$. The PSD of the transmitted signals in $8 \mathrm{~K}$ and $32 \mathrm{~K}$ modes are given in Figs. 3 and 4. With CP-OFDM, the amplitude of the ripple in the useful bandwidth of the PSD of the transmitted signal equal to $10 \log \left(\frac{T_{u}+\Delta}{T_{u}}\right)$ is here negligible due to the low duration of the used guard interval. For the clarity of the representation, the maximum value of each PSD is normalized to $0 \mathrm{~dB}$. As expected, the PSD of the PRTs within the useful bandwidth is $10 \mathrm{~dB}$ higher than the PSD of the DTs. For both modes, the spectral regrowth of the transmitted signal with GCPW before HPA is very close to the original signal. After signal amplification, we can easily notice that the GCPW algorithm reduces the spectral regrowth of the transmitted signal compared to the original signal.

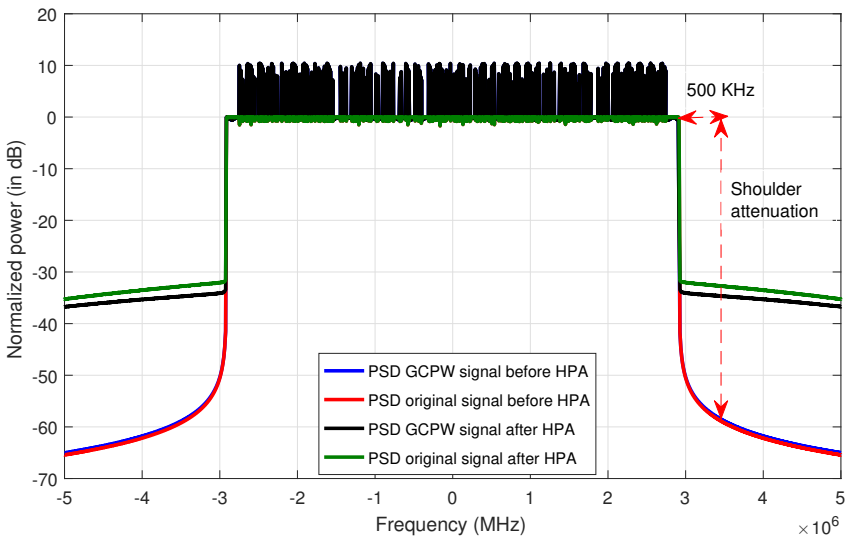

Fig. 4: PSD of the original signal and transmitted signal with GCPW $(G=12, S=200$ ) for: ATSC 3.0, 32K mode, 64 QAM and Rapp model HPA with $p=6$, IBO $=6.4 \mathrm{~dB}$.

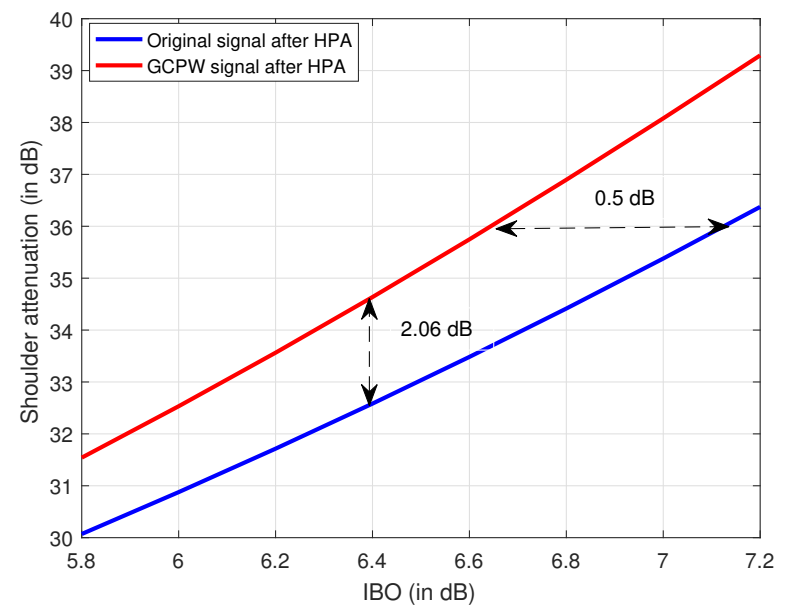

Fig. 5: Shoulder attenuation of original signal and transmitted signal with GCPW $(G=8, S=100)$ for: ATSC 3.0, $8 \mathrm{~K}$ mode, 64 QAM and Rapp model HPA with $p=6$.

\section{Shoulder attenuation}

The shoulder attenuation is classically used to characterize the linearity of the transmitted OFDM signal and then the outof-band distortions without reference to a spectrum mask. It is measured at $500 \mathrm{KHz}$ from the edge carrier as illustrated in Figs. 3 and 4. Note that we don't consider the RF filter whose impact is negligible at $500 \mathrm{KHz}$. Before the HPA, the shoulder attenuation is $52 \mathrm{~dB}$ and $52.36 \mathrm{~dB}$ for the original signal and with GCPW in $8 \mathrm{~K}$ mode, respectively. In $32 \mathrm{~K}$ mode, the shoulder attenuation is $58.14 \mathrm{~dB}$ and $58.53 \mathrm{~dB}$ for the original signal and with GCPW. Fig. 5 shows the shoulder attenuation measurements in function of IBO. We can easily notice that GCPW leads to less spectral regrowth than the original signal for any IBO. At an IBO $=6.4 \mathrm{~dB}$, the GCPW algorithm offers $2.06 \mathrm{~dB}$ gain compared to the original signal. If the targeted shoulder attenuation is fixed at $36 \mathrm{~dB}$, the IBO gain obtained with GCPW is $0.5 \mathrm{~dB}$.

Similarly, in 32K mode the GCPW algorithm leads to less 


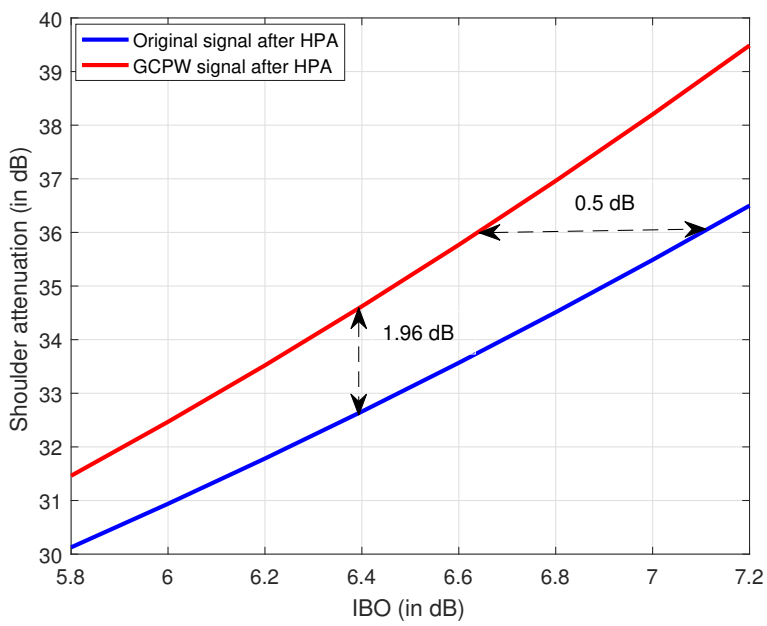

Fig. 6: Shoulder attenuation of original signal and transmitted signal with GCPW $(G=12, S=200)$ for: ATSC 3.0, 32K mode, 64 QAM and Rapp model HPA with $p=6$.

spectral regrowth compared to the original signal for any IBO as illustrated in Fig. 6. The gain at an IBO of $6.4 \mathrm{~dB}$ is 1.96 $\mathrm{dB}$. Also, the IBO gain for a target shoulder measurement of $36 \mathrm{~dB}$ is $0.5 \mathrm{~dB}$.

In Fig. 7, we show the MER and shoulder attenuation gain of GCPW in both $8 \mathrm{~K}$ and $32 \mathrm{~K}$ modes. The MER gain is computed from Figs. 1 and 2 as the difference between the MER of GCPW and the original signal. The shoulder attenuation gain is deduced from Figs. 5 and 6 as the difference between the shoulder attenuation obtained with GCPW and with the original signal. Fig. 7 shows that the evolution of the gain versus the IBO offered by GCPW compared to the original signal is almost the same for the in-band distortions measured by the MER and the out-of-band distortions measured by the shoulder attenuation with however an in-band distortion gain which is higher of $0.5 \mathrm{~dB}$. Furthermore, the MER and the shoulder attenuation gain offered by GCPW is slightly higher in $8 \mathrm{~K}$ mode than in $32 \mathrm{~K}$ mode.

\section{CONCLUSION}

This paper has presented an in-depth study dealing with in-band and out-of-band distortions of ATSC 3.0 transmitters. As any multicarrier communication system, the transmitted signal suffers from high fluctuations. When amplified by NL amplifiers, high PAPR signals are more susceptible to severe distortions. This major disadvantage constrains the HPA to operate in its linear region leading to poor spectral efficiency. PAPR reduction algorithms have been intensively investigated to overcome this issue. In this paper we present a novel TRbased algorithm different from the one proposed for the ATSC 3.0 standard. This algorithm, fully compatible with the ATSC 3.0 standard, named as Grouped Carrier Peak Windowing defines a new kernel targeting the reduction of multiple peaks at each iteration which reduces the total number of iterations. Additionally, a new method to select the highest OFDM signal peaks is also proposed offering less latency complexity and memory requirements. We evaluated through simulations the GCPW algorithm performance in terms of modulation error

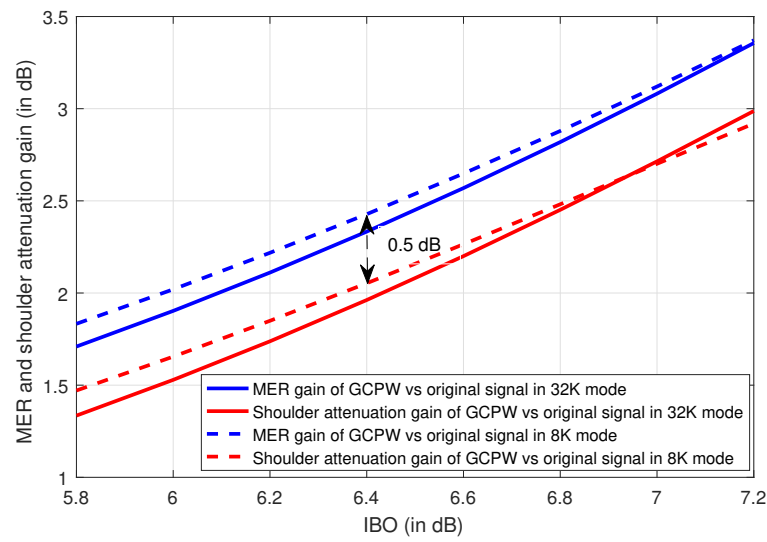

Fig. 7: MER and shoulder attenuation gain of GCPW vs original signal in $8 \mathrm{~K}$ and $32 \mathrm{~K}$ modes.

rate and spectral regrowth in both $8 \mathrm{~K}$ and $32 \mathrm{~K}$ modes. We showed that with the optimized GCPW parameters (which are $G=8$ and $S=100$, and $G=12$ and $S=200$ for $8 \mathrm{~K}$ and $32 \mathrm{~K}$ modes, respectively) the MER gain for an IBO = $6.4 \mathrm{~dB}$ is up to $2.43 \mathrm{~dB}$ and to $2.33 \mathrm{~dB}$ in $8 \mathrm{~K}$ and $32 \mathrm{~K}$ modes, respectively. Additionally, the spectral regrowth of the transmitted signal with GCPW is less than with the original signal for any IBO. With a targeted shoulder attenuation of 36 $\mathrm{dB}$, GCPW offers $0.6 \mathrm{~dB}$ and $0.5 \mathrm{~dB}$ IBO gain in $8 \mathrm{~K}$ and $32 \mathrm{~K}$ modes, respectively. In both modes, we showed that the GCPW algorithm leads to very promising gain in terms of both MER and shoulder attenuation for any IBO. Based on these results and the targeted MER and shoulder attenuation, the IBO and then the operating point of the HPA can be selected.

\section{ACKNOWLEDGMENT}

This work has received a French state support granted to the Convergence TV project through the $20^{r d}$ FUI (transverse inter-ministry funding) program. The authors would also like to thank the "Image \& Réseaux" and "Cap Digital" French business clusters for their support of this work.

\section{REFERENCES}

[1] S. H. Han, and J. H. Lee, "An overview of peak-to-average power ratio reduction techniques for multicarrier transmission," IEEE Trans. Wireless Commun., vol. 12, no. 2, pp. 56-65, Apr. 2005.

[2] O. E. M. J. F.-G. Garcia, and J. M. Pacz-Borallo, "Peak Power Reduction for OFDM Systems With Orthogonal Pilot Sequences," IEEE Trans. Wireless Commun., vol. 5, no. 1, pp. 1536-1276, Jan. 2006.

[3] T. Jiang, and Y. Wu, "An overview: Peak-to-Average Power Ratio Reduction Techniques for OFDM systems," IEEE Trans. on Broadcast., vol. 54, Issue 2, pp. 257-268, June, 2008.

[4] X. Li, and L. J. Cimini, "Effects of clipping and filtering on the performance of OFDM,' IEEE Commun. Lett., vol. 2, no. 5, pp. 131-133, May 1998.

[5] T. A. Wilkinson, and A. E. Jones, "Minimization of the peak-to-mean envelope power ratio of multi-carrier transmission schemes by block coding," In Proc. of IEEE Veh. Technol. Conference (VTC), vol. 2, pp. 825-829, Chicago, Jul. 1995.

[6] S. H. Muller, and J. B. Huber, "OFDM with reduction peak to average power ratio by optimum combination of partial transmit sequences," IEE Electron. Lett., vol. 33, pp. 368-369, Feb. 1997. 
[7] R. W. Bauml, R. F. H. Fischer, and J. B. Huber, "Reducing the peak-toaverage power ratio of multi-carrier modulation by selected mapping," IEE Electron. Lett., vol. 32, no. 22, pp. 2056-2057, Oct. 1996.

[8] J. Tellado, "Peak to Average Ratio Reduction for Multi-carrier Modulation," Ph.D. Thesis, Stanford University, Stanford, CA, USA, 1999.

[9] B. S. Krongold, and D. L. Jones, "PAR reduction in OFDM via active constellation extension," IEEE Trans. Broadcast., vol. 49, issue 2, pp. 258-268, Sept. 2003.

[10] ETSI, "Frame structure channel coding and modulation for a second generation digital terrestrial television broadcasting system (DVB-T2)," ETSI EN 302755 V1.1.1, Sep. 2009.

[11] ATSC 3.0, "ATSC Proposed Standard: Physical Layer Protocol," 2016.

[12] R. Mounzer, M. Crussière, Y. Nasser, and J.-F. Hélard, "Tone reservation based PAPR reduction technique with individual carrier power allocation for multiple peaks reduction," In Proc. of IEEE Veh. Technol. Conference (VTC Spring), Glasgow, Scotland, May 2015.

[13] K. C. Bulusu, M. Crussière, J.-F. Hélard, R. Mounzer, Y. Nasser, O. Rousset, and A. Untersee "Quasi-Optimal Tone Reservation PAPR Reduction Algorithm for Next Generation Broadcasting Systems: A Performance/Complexity/Latency Tradeoff With Testbed Implementation," IEEE Trans. on Broadcasting, pp. 1-7, March 2018.

[14] K. C. Bulusu, M. Crussière, J.-F. Hélard, R. Mounzer, and Y. Nasser, "A Low Latency Algorithm for Efficient PAPR Reduction for DVBT2 and ATSC 3.0 Broadcast," In Proc. of IEEE Internat. Symposium on Broadband Multimedia Systems and Broadcasting (BMSB), Cagliari, Italy, Jun. 2017.

[15] C. Rapp, "Effects of HPA nonlinearity on 4-DPSK-OFDM signal for digital sound broadcasting systems," In Proc. of European Conference on Satellite Communications, pp. 22-24, Liège, Belgium, Oct. 1991.

[16] J. Tellado, and J. Cioffi, "Peak power reduction for multicarrier transmission," In Proc. of IEEE Global Commun. Conference (GLOBECOM), pp. 5-9, Sydney, Australia, Nov. 1998.

[17] S. Zabre, J. Palicot, Y. Louet, and C. Lereau "SOCP approach for OFDM Peak-to-Average Power Ratio reduction in the signal adding context," In Proc. of IEEE Intern. Symposium on Signal Processing and Inf. Technol., Vancouver, BC, Canada, Aug. 2006.

[18] S. Janaaththanan, C. Kasparis, and Barry G. Evans "A gradient based algorithm for PAPR reduction of OFDM using Tone Reservation technique," In Proc. of IEEE Veh. Technol. Conference (VTC-Spring), Singapore, Singapore, May. 2008.

[19] M. Mroué, A. Nafkha, J. Palicot, B. Gavalda, and N. Dagorne, "Performance and Implementation Evaluation of TR PAPR Reduction Methods for DVB-T2," Intern. Journal of Digital Multimedia Broadcasting, vol. 2010, Aug. 2010.

[20] M. Mroué, A. Nafkha, and J. Palicot, "An innovative low complexity PAPR reduction TR-based technique for DVB-T2 system," In Proc. of IEEE Intern. Congress on Ultra Modern Telecommun. and Control Systems and Workshops, Moscow, Russia, Oct. 2010. 